\title{
Recent results on heavy-ion induced reactions of interest for neutrinoless double beta decay at INFN-LNS
}

\author{
Manuela Cavallaro ${ }^{1 *}$, Luis Acosta ${ }^{2}$, Clementina Agodi ${ }^{1}$, Carmen Altana ${ }^{1}$, Paulina Amador-Valenzuela ${ }^{3}$, Naftali \\ Auerbach $^{4}$, Jose Barea ${ }^{5}$, Jessica I. Bellone ${ }^{1}$, Roelof Bijker ${ }^{6}$, Danilo Bonanno ${ }^{7}$, Thereza Borello-Lewin ${ }^{8}$, Ismail Boztosun ${ }^{9}$, \\ Vincenzo Branchina ${ }^{1,7}$, Sandro Brasolin ${ }^{10}$, Giuseppe A. Brischetto ${ }^{1,11,13}$, Oscar Brunasso ${ }^{10}$, Stefano Burrello ${ }^{1}$, Salvatore \\ Calabrese $^{1,2}$, Luciano Calabretta ${ }^{1}$, Daniela Calvo ${ }^{10}$, Vittoria Capirossi ${ }^{10,12}$, Francesco Cappuzzello $^{1,11}$, Diana Carbone $^{1}$, \\ Luis E. Charon Garcia ${ }^{2}$, Efrain R. Chávez Lomeli ${ }^{2}$, Ruofu Chen ${ }^{14}$, Irene Ciraldo ${ }^{1,11}$, Maria Colonna ${ }^{1}$, Grazia D'Agostino $^{1}$, \\ Franck Delaunay ${ }^{10,12,15}$, Nikit Deshmukh ${ }^{16}$, Haris Djapo ${ }^{9}$, Gianluigi De Geronimo ${ }^{17}$, K. De Los Rìos ${ }^{2}$, Carlo Ferraresi ${ }^{18}$, \\ Jonas L. Ferreira ${ }^{19}$, Jacopo Ferretti ${ }^{20,21}$, Paolo Finocchiaro ${ }^{1}$, Suna Firat ${ }^{9}$, Maria Fisichella ${ }^{10}$, Antonino Foti $^{7}$, Giuseppe \\ Gallo $^{1,11}$, Hugo Garcia-Tecocoatzi ${ }^{20,22}$ Aylin Hacisalihoglu ${ }^{23}$, Arcadio Huerta-Hernandez ${ }^{2}$, Jenni Kotila ${ }^{24}$, Yasemin \\ Kucuk $^{9}$, Felice Iazzi ${ }^{10,12}$, Gaetano Lanzalone ${ }^{1,25}$, José Antonio Lay ${ }^{26}$, Laura La Fauci ${ }^{1,11}$, Francesco La Via ${ }^{27}$, Horst \\ Lenske $^{28}$, Roberto Linares ${ }^{19}$, Domenico Lo Presti ${ }^{11,7}$, Jesus Lubian ${ }^{19}$, Junbing Ma ${ }^{14}$, Daniel Marin-Lámbarri ${ }^{2}, J$. Mas $^{2}$ \\ Ruiz $^{2}$, Nilberto H. Medina ${ }^{8}$, Djalma R. Mendes ${ }^{19}$, Paolo Mereu ${ }^{10}$, Mauricio Moralles ${ }^{29}$, Lorenzo Neri ${ }^{1}$, José R. B. \\ Oliveira $^{8}$, Athena Pakou $^{30}$, Luciano Pandola ${ }^{1}$, Horia Petrascu ${ }^{31}$, Norbert Pietralla ${ }^{32}$, Federico Pinna ${ }^{10,12}$, Santo Reito ${ }^{7}$, \\ Philipp C. Ries ${ }^{32}$, Marcia R. D. Rodrigues ${ }^{8}$, Antonio D. Russo ${ }^{1}$, Giuseppe Russo ${ }^{7,11}$, Elena Santopinto ${ }^{20}$, Roberto B. B. \\ Santos $^{33}$, Leonardo Serbina ${ }^{31}$, Onoufrios Sgouros ${ }^{1}$, Marcilei A. G. da Silveira ${ }^{33}$, Selçuk O. Solakci ${ }^{9}$, George Souliotis ${ }^{34}$, \\ Vasileios Soukeras ${ }^{1}$, Alessandro Spatafora ${ }^{1,11}$, Domenico Torresi ${ }^{1}$, Salvatore Tudisco ${ }^{1}$, Hesiquio Vargas Hernandez ${ }^{2}$, \\ Ruslan I. M. Vsevolodovna ${ }^{20,22}$, Jan Song Wang ${ }^{14}$, Volker Werner ${ }^{32}$, Yan Yun Yang ${ }^{14}$, Aydin Yildirin ${ }^{9}$, Vinicius A. B. \\ Zagatto $^{8}$
}

for the NUMEN Collaboration

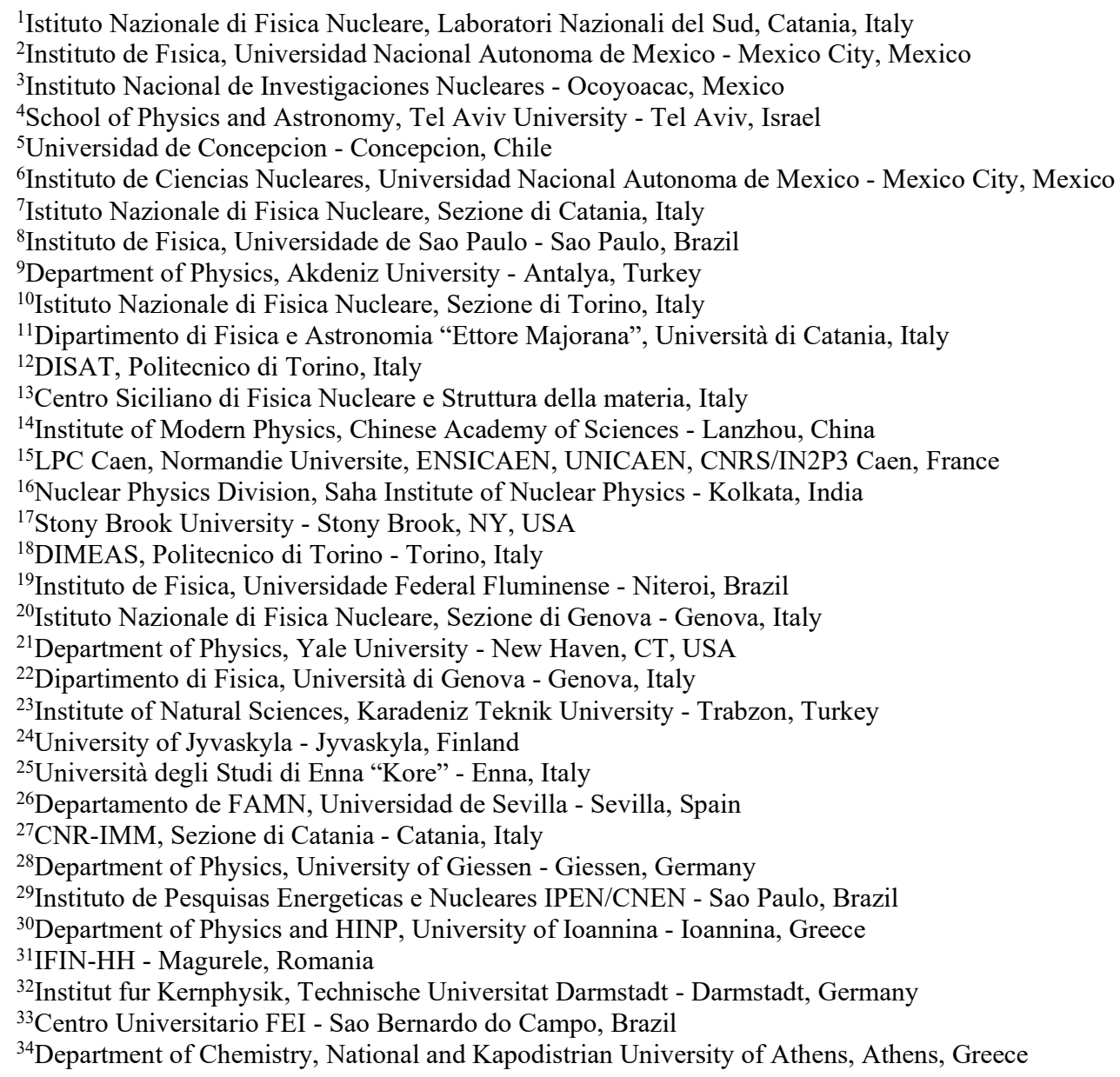

*Corresponding author: manuela.cavallaro@lns.infn.it 


\begin{abstract}
The possibility to use a special class of heavy-ion induced direct reactions, such as double charge exchange reactions, is discussed in view of their application to extract information that may be helpful to determinate the nuclear matrix elements entering in the expression of neutrinoless double beta decay halflife. The methodology of the experimental campaign presently running at INFN - Laboratori Nazionali del Sud is reported and the experimental challenges characterizing such activity are described.
\end{abstract}

\section{Introduction}

Charge exchange reactions are versatile tools for both reaction and nuclear structure studies. An especially appealing aspect is the possibility to use them to investigate the spin-isospin-dependent response of nuclei. These studies have a genuine nuclear physics interest, since they reflects a key aspect of nuclear dynamics. However, beyond that, there is an even broader impact of such investigations because of their relevance for nuclear beta decay, thus connecting strong and weak interactions. In this context, the NUMEN and NURE projects at LNS Catania [1-3] aim to promote heavy-ion induced single and double charge exchange reactions in a systematic approach, with the intention to extract information on nuclear matrix elements for single and double charge exchange processes and to identify the possible connections with double beta decay.

In the present paper, the strategy and methodologies of the above-mentioned programmes, the challenges of the experimental activities together with the status of the already performed experimental runs are described and discussed.

\section{The motivation}

The neutrino is a key particle for astrophysics, nuclear physics, particle physics and cosmology. It is an elementary particle that has only a weak charge, no electric and no colour charge. Thus, neutrino interactions with nuclei are extremely weak and experimental studies related to neutrinos are difficult.

Neutrinos have been extensively studied experimentally and theoretically in the recent 4-5 decades, but some of their fundamental properties are still not well understood and several basic questions about neutrinos remain yet unsolved. Among them, the nature of the neutrino, i.e. whether it is a Majorana particle (neutrino $=$ antineutrino) or a Dirac particle (neutrino $\neq$ antineutrino) and the absolute mass scale. These questions can be studied well by investigating neutrino-related weak processes in nuclei, such as neutrinoless double beta decays $(0 v \beta \beta)$. If detected, $0 v \beta \beta$ would provide evidence for the Majorana nature of the neutrino and the non-zero mass [4-9].

The $0 v \beta \beta$ Nuclear Matrix Elements (NMEs) are very sensitive to nuclear physics involved in $0 v \beta \beta$ probing the nuclear wave functions of the initial and final states, the spin-isospin correlations, nuclear structure models, nuclear medium effects, the renormalization (quenching) of the effective weak coupling in nuclei, and so on. However, there are no experimental methods to directly measure $0 v \beta \beta$ NMEs and accurate theoretical calculations of them are hard, leading to discrepancy factors larger than two, as presently reported in literature. Thus experimental inputs relevant to the $0 v \beta \beta$ responses are useful to help evaluate the $0 v \beta \beta$ NMEs and to constrain the calculations.

In this context, an experimental campaign [1] has started at the INFN-Laboratori Nazionali del Sud in Catania using the MAGNEX large acceptance magnetic spectrometer [10]. It focuses on the study of heavy-ion induced Double Charge Exchange (DCE) reactions and competing reaction channels involving the nuclei of interest for $0 v \beta \beta$ decay.

The possible connections of DCE measured cross sections with double beta decay NMEs and consequently with the still unsolved neutrino puzzles are among the goals of the present studies.

\section{Heavy ion induced double charge exchange reactions}

Important experimental challenges must be addressed to measure heavy ion induced DCE reactions.

Such challenges are related to the request to detect heavy ions with good isotopic separation and energy resolution in a wide angular range, including zero-degree, in order to distinguish transitions to individual states and explore a wide momentum transfer range.

In addition, the rather tiny DCE cross sections (down to few $\mathrm{nb}$ ) demand for a challengingly high experimental sensitivity, which strongly depend on the rejection capability against unwanted events. These latter may be generated by competing reaction processes, which are rather likely to occur in view of the small DCE cross sections. For this reason high resolution particle identification is a necessary prerequisite for the experiment. In addition, spurious data associated to a wrong determination of detection parameters could casually be misinterpreted as good DCE data. Such background sources can be subtle and require stringent conditions for the experimental set-up together with a very careful characterization of the detection response in order to get rid of and gain the necessary sensitivity for performing meaningful DCE investigations [11].

These results are pursued thanks to the use of the MAGNEX spectrometer, a tool with high performance and flexibility (see Section 4) [12-15].

The accurate description of reactions involving complex nuclei is a demanding task also for nuclear theory. From the side of reaction theory initial and final state interactions of two many-body systems have to be under control and the transition amplitude must be described properly. Reactions involving a few selected degrees of freedom, keeping the bulk of projectile and target essentially intact are the most appropriate tools for 
those investigations and direct reaction theory can provide the suitable toolbox.

Nuclear structure theory is asked to provide accurate input for the spectroscopy of both projectile and target, even if the main interest is to explore the spectrum of one of the reaction partners only.

Despite of these caveats coherent ion-ion interactions have been used quite successfully in the past to investigate excitations in the quasi-elastic region. Moreover new theoretical developments for the description of the reaction mechanism involved in heavy ion induced reactions have been achieved under specific conditions and others are presently in progress within the NUMEN project [16-20].

\section{The experiments}

The experimental challenges mentioned in the previous section are faced at INFN-LNS by using the K800 Superconducting Cyclotron to accelerate beams and the MAGNEX large acceptance magnetic spectrometer for the detection of the ejectiles.

The MAGNEX spectrometer is a large acceptance magnetic device made up of a large aperture vertically focusing quadrupole and a horizontally bending dipole magnet. MAGNEX was designed to investigate processes characterized by very low yields and allows the identification of heavy ions with quite high mass $(\Delta \mathrm{A} / \mathrm{A} \sim 1 / 160)$, angle $\left(\Delta \theta \sim 0.2^{\circ}\right)$ and energy resolutions $(\Delta \mathrm{E} / \mathrm{E} \sim 1 / 1000)$, within a large solid angle $(\Omega \sim 50 \mathrm{msr})$ and momentum range $(-14 \%<\Delta \mathrm{p} / \mathrm{p}<+10 \%)$. Highresolution measurements for quasi-elastic processes, characterized by differential cross sections falling down to tens of $\mathrm{nb} / \mathrm{sr}$, were already performed by this setup [2126]. A crucial feature is the implementation of a technique of trajectory reconstruction, based on differential algebraic techniques, which allows solving the equation of motion of each detected particle to $10^{\text {th }}$ order [27-29]. This is a unique characteristic of MAGNEX, which guarantees the above-mentioned performances and its relevance in the worldwide scenario of heavy-ion physics.

The experimental activity with accelerated beams proposed and presently in progress consists of two main classes of experiments, corresponding to the exploration of the two directions of isospin lowering $\tau^{-} \tau^{-}$and rising $\tau^{+} \tau^{+}$, characteristic of $\beta^{-} \beta^{-}$and $\beta^{+} \beta^{+}$decays, respectively. In particular, the $\beta^{+} \beta^{+}$direction in the target is investigated using an ${ }^{18} \mathrm{O}^{8+}$ beam and measuring the $\left({ }^{18} \mathrm{O},{ }^{18} \mathrm{Ne}\right) \mathrm{DCE}$ transitions, together with other reaction channels involving same beam and target. Similarly, the $\beta^{-} \beta^{-}$ direction is explored via the $\left({ }^{20} \mathrm{Ne},{ }^{20} \mathrm{O}\right)$ reaction, using a ${ }^{20} \mathrm{Ne}^{10+}$ beam and detecting the reaction products of the DCE channel and of the other open channels characterized by same projectile and target.

Exploratory investigations of the two classes of experiments have been already performed, highlighting the strengths and the limiting aspects of the adopted technique and establishing the best working conditions [30-34].

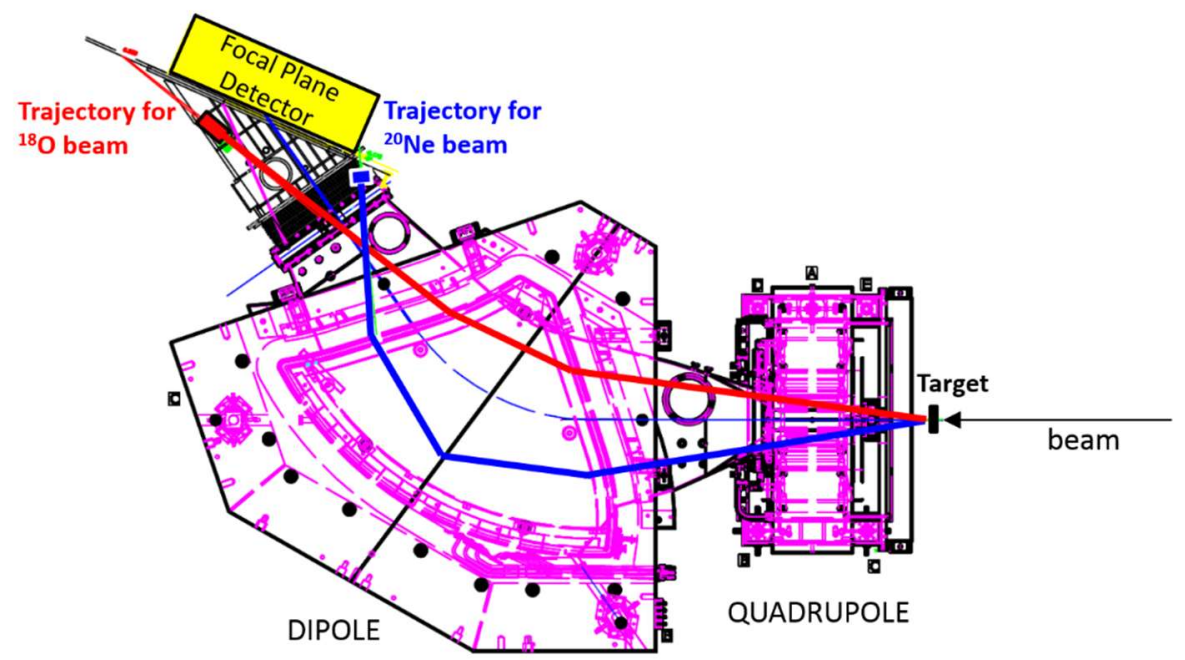

Fig. 1. Drawing of the MAGNEX spectrometer showing two typical trajectories of the ${ }^{18} \mathrm{O}$ and ${ }^{20} \mathrm{Ne}$ beams in the experimental conditions used for the DCE measurements.

\subsection{Experiments with ${ }^{18} \mathrm{O}$ beam $\left(\beta^{+} \beta^{+}\right.$direction)}

For the experiments of this class, the reaction channels of our interest are listed below:

- Elastic and inelastic scattering $\left({ }^{18} \mathrm{O},{ }^{18} \mathrm{O}\right)$

- DCE reaction $\left({ }^{18} \mathrm{O},{ }^{18} \mathrm{Ne}\right)$

- Charge-exchange reaction $\left({ }^{18} \mathrm{O},{ }^{18} \mathrm{~F}\right)$
- Two-proton pickup reaction $\left({ }^{18} \mathrm{O},{ }^{20} \mathrm{Ne}\right)$

- One-proton pickup reaction $\left({ }^{18} \mathrm{O},{ }^{19} \mathrm{~F}\right)$

- Two-neutron stripping reaction $\left({ }^{18} \mathrm{O},{ }^{16} \mathrm{O}\right)$

- One-neutron stripping reaction $\left({ }^{18} \mathrm{O},{ }^{17} \mathrm{O}\right)$

One of the main challenges of such experiments is the measurement at very forward angles, including zerodegree. This is performed by placing the spectrometer with its optical axis at $+3^{\circ}$ with respect to the beam axis. Thanks to its large angular acceptance, a range $-2^{\circ}<\theta_{\text {lab }}$ $<+9^{\circ}$ is thus covered. The MAGNEX quadrupole and 
dipole magnetic fields are set in order that the incident beam, after passing through the magnets, reaches a region besides the FPD. For this class of experiments, in fact, the incident beam $\left({ }^{18} \mathrm{O}^{8+}\right)$ has higher magnetic rigidity $(\mathrm{B} \rho)$ than the ones of the ejectiles of interest (namely ${ }^{18} \mathrm{Ne},{ }^{18} \mathrm{~F}$, ${ }^{20} \mathrm{Ne},{ }^{19} \mathrm{~F},{ }^{16} \mathrm{O},{ }^{17} \mathrm{O}$ with maximum charge state). The beam stops in a specifically designed Faraday Cup, which measures the incident charge in each run, placed in the high-B $\rho$ region besides the FPD.

A drawing of the MAGNEX spectrometer and an example of the typical ${ }^{18} \mathrm{O}$ beam trajectory inside the spectrometer is shown in Fig. 1.

${ }^{116} \mathrm{Sn},{ }^{76} \mathrm{Se}$ and ${ }^{48} \mathrm{Ti}$ are the targets already explored via $\left({ }^{18} \mathrm{O},{ }^{18} \mathrm{Ne}\right)$ reaction at 15 and $22 \mathrm{AMeV}$ in order to study the ${ }^{116} \mathrm{Sn} \rightarrow{ }^{116} \mathrm{Cd},{ }^{76} \mathrm{Se} \rightarrow{ }^{76} \mathrm{Ge}$ and ${ }^{48} \mathrm{Ti} \rightarrow{ }^{48} \mathrm{Ca}$ transitions, respectively, and the competing channels as shown in Fig. 2. The reduction and analysis of the collected data is presently in progress.

\subsection{Experiments with ${ }^{20} \mathrm{Ne}$ beam $\left(\beta^{-} \beta^{-}\right.$direction)}

In the class of experiments with ${ }^{20} \mathrm{Ne}^{10+}$ beams, the reaction channels we are interested are the following:

- Elastic and inelastic scattering $\left({ }^{20} \mathrm{Ne},{ }^{20} \mathrm{Ne}\right)$

- DCE reaction $\left({ }^{20} \mathrm{Ne},{ }^{20} \mathrm{O}\right)$

- Charge Exchange reaction $\left({ }^{20} \mathrm{Ne},{ }^{20} \mathrm{~F}\right)$

- Two-proton stripping reaction $\left({ }^{20} \mathrm{Ne},{ }^{18} \mathrm{O}\right)$

- One-proton stripping reaction $\left({ }^{20} \mathrm{Ne},{ }^{19} \mathrm{~F}\right)$

- Two-neutron pickup reaction $\left({ }^{20} \mathrm{Ne},{ }^{22} \mathrm{Ne}\right)$

- One-neutron pickup reaction $\left({ }^{20} \mathrm{Ne},{ }^{21} \mathrm{Ne}\right)$

For these experiments, the incident beam $\left({ }^{20} \mathrm{Ne}^{10+}\right)$ has a lower magnetic rigidity with respect to the reaction ejectiles of interest. Thus, for a fixed magnetic field setting, the beam will be more bent than the ejectiles of interest. The spectrometer optical axis is typically placed at $-3^{\circ}$, thus the covered angular range is $-8^{\circ}<\theta_{\text {lab }}<+3^{\circ}$. The quadrupole and dipole magnetic fields of MAGNEX are set in order that the ${ }^{20} \mathrm{Ne}^{10+}$ beam reaches the low-B $\rho$ region besides the FPD.

A peculiarity of these experiments concerns the treatment of the different charge states of the beam emerging out of the target. The beam components characterized by charge states lower than $10^{+}$, mainly ${ }^{20} \mathrm{Ne}^{9+}$ and ${ }^{20} \mathrm{Ne}^{8+}$, produced by the interaction of the beam with the electrons of the target material, have a magnetic rigidity which is similar to the ions of interest. Therefore, they enter in the FPD acceptance, causing a limitation in the rate tolerable by the detector.

In order to stop these unwanted ${ }^{20} \mathrm{Ne}$ particles, two aluminium shields are mounted upstream the sensitive region of the focal plane detector.

The shields act on a limited phase space region which stops the $9^{+}$and $8^{+}$beams and elastic scattering at very forward angles, but not the other reaction channels generated by these beams. It is known that the charge state distribution of a heavy-ion beam after crossing a material depends on the bombarding energy and on the chemical composition of the target. The targets relevant for NUMEN generate an unwanted charge distribution that can be conveniently changed, minimizing the amount of ${ }^{20} \mathrm{Ne}^{9+}$ and ${ }^{20} \mathrm{Ne}^{8+}$, by adding an appropriate second foil (post-stripper) downstream of the isotopic target. Recently a specific study of different materials to be used as poststripper has been performed [33].

The systems already experimentally explored using the $\left({ }^{20} \mathrm{Ne},{ }^{20} \mathrm{O}\right)$ reaction at $15 \mathrm{AMeV}$ are the ${ }^{116} \mathrm{Cd}$ target (to study the ${ }^{116} \mathrm{Cd} \rightarrow{ }^{116} \mathrm{Sn}$ transition), the ${ }^{130} \mathrm{Te}$ (for the ${ }^{130} \mathrm{Te} \rightarrow{ }^{130} \mathrm{Xe}$ ) and the ${ }^{76} \mathrm{Ge}$ (for the ${ }^{76} \mathrm{Ge} \rightarrow{ }^{76} \mathrm{Se}$ ). The data reduction and analysis is in progress.

A scheme of the transitions already studied in the experimental runs is shown in Fig. 2.
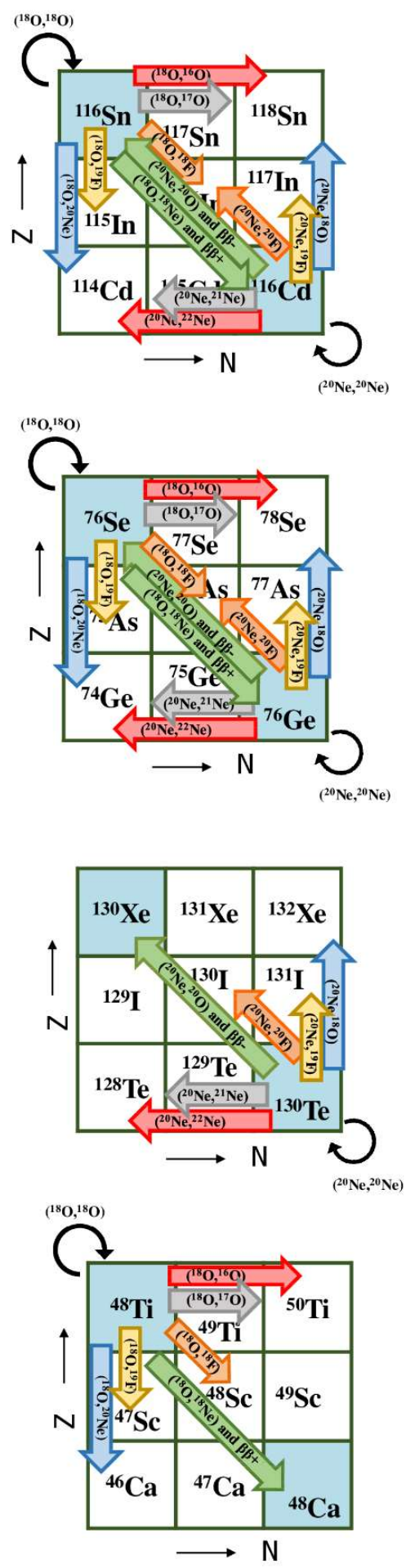

Fig. 2. Scheme of the reaction channels already studied within the NURE and NUMEN experiments. 


\section{Conclusions}

A systematic study of heavy-ion induced double charge exchange reactions and of the other reaction channels characterized by the same projectile and target is in progress at INFN-LNS. The main goal is to investigate the nuclear response to DCE reactions for all the isotopes candidate for $0 v \beta \beta$ decay to give experimentally driven indication towards the determination of $0 v \beta \beta$ NMEs.

In the present paper, the focus is on the techniques adopted to set up the MAGNEX spectrometer for the challenging measurements of such suppressed reaction channels. The strategy used to perform the zero-degree measurement, based on an accurate simulation of the ion trajectories along the spectrometer and on the use of two Faraday cups properly designed for each category of experiments, is described.

\section{Acknowledgments}

This project has received funding from the European Research Council (ERC) under the European Union's Horizon 2020 research and innovation programme (grant agreement No 714625).

\section{References}

1. F. Cappuzzello et al., Eur. Phys. J. A 54, 72 (2018)

2. M. Cavallaro et al., Proceedings of Science, PoS(BORMIO2017) 015 (2017)

3. F. Cappuzzello et al., J. Phys.: Conf. Ser. 630, 012018 (2015)

4. H. Ejiri, J. Suhonen, K. Zuber, Physics Reports 797, 1 (2019)

5. J. Vergados, H. Ejiri, F. Šimkovic, Rep. Progr. Phys. 75, 106301 (2012)

6. J. Suhonen, O. Civitarese, J. Phys. G: Nucl. Part. Phys. 39, 124005 (2012)

7. S. Dell'Oro, S. Marcocci, and F. Vissani, Phys. Rev. D 90, 033005 (2014)

8. J. Engel, J. Menéndez, Rep. Progr. Phys. 60, 046301 (2017)

9. P. Vogel, Jour. of Phys. G: Nucl. and Part. Phys., 39 124002 (2012)

10. F. Cappuzzello et al., Eur. Phys. J. A 51,145 (2015)

11. S. Calabrese et al., in preparation.

12. F. Cappuzzello et al. Eur. Phys. J. A 52, 167 (2016)

13. C. Boiano et al., IEEE Trans. on Nucl. Science 4723837, 3563 (2008)

14. M. Cavallaro et al., Nucl. Instr. and Meth. A 700, 65 (2013)

15. M. Cavallaro et al. Phys. Rev. C 93, 064323 (2016)

16. H. Lenske et al., Phys. Rev. C 98, 044620 (2018)

17. E. Santopinto et al., Phys. Rev. C 98 061601(R) (2018)

18. H. Lenske et al., Prog. Part. Nucl. Phys. 109, 103716 (2019)
19. J.I. Bellone et al., J. Phys. Conf. Ser. 1056, 012004 (2018)

20. J.A. Lay et al., J. Phys. Conf. Ser. 1056, 012029 (2018)

21. F. Cappuzzello et al., Eur. Phys. J. A 52, 169 (2016)

22. D. Carbone et al., Phys. Rev. C 95, 034603 (2017)

23. V.A.B. Zagatto et al., Phys. Rev. C 97, 054608 (2018)

24. M. J. Ermamatov et al., Phys. Rev. C 94, 024610 (2016)

25. J.R.B. Oliveira et al., Journal of Physics G: Nuclear and Particle Physics 40, 105101 (2013)

26. F. Cappuzzello et al., Nucl. Instr. and Meth. A 763, 314 (2014)

27. F. Cappuzzello, et al., Nucl. Instr. and Meth. A 638, 74 (2011)

28. A. Lazzaro et al., Nucl. Instr. and Meth. A 585, 136 (2008)

29. A. Lazzaro et al., Nucl. Instr. and Meth. A 602, 494 (2009)

30. F. Cappuzzello et al., Eur. Phys. J. A 51, 145 (2015)

31. S. Calabrese et al., Acta Phys. Pol. B 49, 275 (2018)

32. M. Cavallaro et al., Nucl. Instr. and Meth. B (in press) https://doi.org/10.1016/j.nimb.2019.04.069.

33. M. Cavallaro et al., Results in Phys. 13, 102191 (2019)

34. A. Spatafora et al., Phys. Rev. C 100, 034620 (2019) 